\title{
FLoRÍSTICA E CARACTERIZAÇÃo dOS CAMPOS RUPESTRES SOBRE CANGa na Serra da Calçada, Minas Gerais, Brasil ${ }^{1}$
}

\author{
Pedro Lage Viana ${ }^{2} \&$ Julio Antonio Lombardi ${ }^{3}$
}

\section{Resumo}

(Florística e caracterização dos campos rupestres sobre canga na Serra da Calçada, Minas Gerais, Brasil) Os campos rupestres sobre canga são um ambiente peculiar e freqüente na região do Quadrilátrero Ferrífero, Minas Gerais. Sua vegetação, associada a afloramentos hematíticos, apresenta uma série de adaptações morfológicas e fiosiológicas, assim como alto grau de endemismo. Foi realizado um leventamento florístico, restrito às fanerógamas, e uma caracterização descritiva dos campos rupestres sobre canga na Serra da Calçada. Para tal, realizaram-se campanhas de campo mensais, entre junho de 2001 e agosto de 2002, e foram analisados exemplares depositados no herbário BHCB. O levantamento contou com 358 espécies distribuídas em 70 famílias, sendo as famílias mais ricas Poaceae (43 spp.), Asteraceae (42 spp.), Fabaceae e Myrtaceae (21 spp. cada), Melastomataceae e Orchidaceae (18 spp. cada). A maioria das espécies ( 210 spp.) ocorre em canga nodular, 153 em canga couraçada, 93 em capões de mata e $47 \mathrm{em}$ áreas antropizadas. $\mathrm{O}$ alto grau de ameaça a que este ambiente está submetido e a presença expressiva de espécies ameaçadas de extinção implicam na necessidade urgente de preservação de áreas que compreendem campos rupestres sobre canga.

Palavras chave: flora, campos rupestres, canga, campos ferruginosos, Quadrilátero Ferrífero.

\section{Abstract}

(Floristics and characterization of the ferrugineous rocky grasslands at Serra da Calçada, Minas Gerais state, Brazil) Ferrugineous rocky grasslands are a peculiar environment frequently found at the "Quadrilátero Ferrifero" region, Minas Gerais State. The vegetation, associated to hematitic outcrops, presents physiological and morphological adaptations, as well as high levels of endemism. A floristic survey, restricted to phanerogames, and a descriptive characterization of the ferrugineous rocky grasslands of Serra da Calçada are presented. Studies of specimens collected from July 2001 and August 2002 were complemented by exame of collections deposited at BHCB Herbarium. A total of 358 species distributed in 70 families were registred. The richest families were Poaceae (43 spp.), Asteraceae (42 spp.), Fabaceae and Myrtaceae (21 spp. each), Melastomataceae and Orchidaceae (18 spp. each). Most of the species (210 spp.) occur on grassy fields (or "canga nodular"), while 153 occur on rocky fields (or "canga couraçada"), 93 on forest islands and 47 on anthropized areas. Ferrugineus grasslands is under threatened due to mining exploration and urban development, therefore urgent conservation measures are essential to protect threatened plant species.

Key words: flora, rocky grasslands, "canga”, ferrugineous grasslands, Quadrilátero Ferrífero.

\section{INTRODUÇÃO}

A Serra do Espinhaço é uma cadeia montanhosa que atravessa de norte a sul os estados da Bahia e de Minas Gerais. É formada por dois blocos principais, a Chapada Diamantina, no estado da Bahia, e a cadeia do Espinhaço, em Minas Gerais (Giulietti et al. 1997, Harley 1995). As quotas altitudinais variam aproximadamente entre 700 e $2000 \mathrm{~m}$ acima do nível do mar e seu ambiente montanhoso representa um centro de concentração de biodiversidade e endemismos (Harley 1995).

Ao longo da Serra do Espinhaço, em altitudes acima de aproximadamente $900 \mathrm{~m}$, a vegetação dos campos rupestres se destaca na

Artigo recebido em 06/2006. Aceito para publicação em 11/2006.

${ }^{1}$ Parte da Monografia de Bacharelado em Ciências Biológicas do primeiro autor, pela Universidade Federal de Minas Gerais ${ }^{2}$ Universidade Federal de Juiz de Fora, Instituto de Ciências Biológicas, Departamento de Botânica, 36036-900, Juiz de Fora, MG, Brasil. vianapl@yahoo.com.br

${ }^{3}$ Universidade Estadual Paulista Júlio de Mesquita Filho, Instituto de Biociências de Rio Claro, Departamento de Botânica, 13506-900, Rio Claro, SP, Brasil. 
composição da paisagem. O termo 'campo rupestre' foi primeiramente utilizado por Magalhães (1966) para designar o tipo de vegetação associada a afloramentos quartzíticos, tão característica na Serra do Espinhaço. Este termo vem sendo amplamente utilizado por grande parte dos pesquisadores envolvidos no estudo da vegetação na Serra do Espinhaço (Giulietti et al. 2000, Giulietti et al. 1987, Giulietti et al. 1997, Harley 1995, Meguro et al. 1994, Pirani et al. 1994, Pirani et al. 2003, Zappi et al. 2003). Campos rupestres sobre rochas quartzíticas ocorrem, de forma disjunta, fora das abrangências da Serra do Espinhaço, como na Serra de Ibitipoca (Rodela 1998) e Serra da Canastra (Nakajima \& Semir 2001, Romero \& Martins 2001) em Minas Gerais, e na região da Chapada dos Veadeiros e Serra dos Pirineus, Goiás (Mendonça et al. 1998).

Rizzini (1979) inclui também a vegetação associada a afloramentos hematíticos, comum na região do Quadrilátero Ferrífero, Minas Gerais, e na Serra de Carajás, Pará, no conceito de campos rupestres, denominando-os como campos ferruginosos, termo também utilizado por Vincent et al. (2002) e Vincent (2004). Essa formação vegetal também é referida como vegetação de canga (Morelato \& Rosa 1991, Secco \& Mesquita 1983, Silva 1991, Silva 1992).

Os campos rupestres sobre canga constituem um ambiente caracteristicamente adverso ao estabelecimento de plantas (Vincent et al. 2002). Alguns fatores limitantes, como alta incidência solar, pouca matéria orgânica disponível e solo com altas concentrações de metais pesados (Teixeira \& Lemos-Filho 2002, Vincent et al. 2002), podem ser considerados relevantes na biologia e no processo evolutivo de espécies que ocorrem neste ambiente. Muitas espécies apresentam adaptações morfológicas e/ou fisiológicas a ambientes xerofíticos, tais como folhas coriáceas ou suculentas, modificações de órgãos em estruturas de reserva, presença de pilosidade densa nas folhas e ramos, e até anatomia Kranz, presente em espécies com metabolismo CAM (Larcher 1995). Em função dos altos teores de metais pesados no solo, as plantas podem apresentar ecótipos com nanismo ou gigantismo (Porto \& Silva 1989) e alta capacidade de reter metais pesados em seus tecidos (Teixeira \& Lemos-Filho 1998, Vincent 2004).

Porto \& Silva (1989) e Silva (1991) mencionam baixa diversidade vegetal e ocorrência frequiente de espécies endêmicas em substratos metalíferos. Inventários realizados em formações campestres que incluem campos rupestres sobre canga no Quadrilátero Ferrífero (Brandão 1992, Brandão \& Gavilanes 1990, Brandão et al. 1991, Grandi et al. 1988), em geral, indicam valores de riqueza florística menores que em outras áreas da Serra do Espinhaço (Giulietti et al. 1987, Harley 1995, Harley \& Mayo 1980, Pirani et al. 1994, Pirani et al. 2003, Zappi et al. 2003).

Segundo Drummond et al. (2005) o Quadrilátero Ferrífero é uma área prioritária para a conservação da biodiversidade no estado de Minas Gerais, de importância biológica extrema. Cabe ressaltar o alarmante grau de ameaça a que estão submetidos os campos rupestres sobre canga do Quadrilátero Ferrífero. A região, juntamente com a Serra dos Carajás, no Pará, compreende aproximadamente $98 \%$ das jazidas de minério de ferro do Brasil, sendo que a maior parte é explorada no Quadrilátero Ferrífero (BRASIL 1978). Grandes extensões deste ambiente já foram completamente eliminadas por atividades mineradoras e quase a totalidade dos remanescentes pertence a empresas de mineração ou são áreas fortemente afetadas pela expansão imobiliária. Apenas uma unidade de conservação em Minas Gerais, o Parque Estadual da Serra do Rola Moça, possui pequenas porções de campos rupestres sobre canga, área insuficiente para presevar a diversidade deste ambiente peculiar.

O objetivo deste trabalho é contribuir para o conhecimento dos campos rupestres sobre canga, fornecendo uma listagem de espécies de fanerógamas e uma análise da sua composição florística na Serra da Calçada, Minas Gerais. 


\section{Material e Métodos}

A Serra da Calçada se situa no Quadrilátero Ferrífero, extremo norte da Serra da Moeda, município de Brumadinho e segue aproximadamente $8 \mathrm{~km}$ em direção sul, a partir do ponto $20^{\circ} 05^{\prime} 35^{\prime \prime} \mathrm{S}$, $43^{\circ} 59^{\prime} 01^{\prime \prime} \mathrm{W}$, onde se localiza o condomínio residencial Retiro das Pedras. São encontrados, dentre os tipos vegetacionais na região, matas de galeria, capões de mata, campos rupestres sobre quartzito e campos rupestres sobre canga. Estes últimos ocupam, na área de estudo, pouco mais de 500 ha. As quotas altitudinais variam de aproximadamente 900 a $1426 \mathrm{~m}$.

As coletas se restringiram aos campos rupestres sobre canga. Consideraram-se campos rupestres sobre canga a vegetação sobre solo hematítico, podendo ser sobre conglomerados maciços (canga couraçada sensu Rizzini (1979)) ou sobre solo pedregoso, que possibilita o estabelecimento massivo de vegetação graminóide (canga nodular sensu Rizzini (1979)). Pequenos capões de vegetação sub-arbórea densa, associados aos campos rupestres sobre canga, assim como áreas com visível ação antrópica, também foram amostrados. Foram, portanto, consideradas quatro fitofisionomias distintas como parte do complexo campos rupestres sobre canga, para análise da vegetação: canga couraçada, canga nodular, capões de mata e áreas antrópicas.

Realizaram-se campanhas de campo mensais entre junho de 2001 e agosto de 2002. Para compor a lista de espécies foram realizadas coletas aleatórias e restritas a espécies fanerógamas em estado fértil. Todo o material coletado encontra-se depositado no herbário da Universidade Federal de Minas Gerais (BHCB). Foram, também, examinados exemplares previamente depositados nesse herbário, coletados na presente área de estudo e com devida especificação do solo ferruginoso em seus rótulos.

A identificação das espécies se deu por meio de bibliografia específica, comparação com exsicatas determinadas depositadas no Herbário do Departamento de Botânica do Instituto de Ciências Biológicas da Universidade Federal de Minas Gerais (Herbário BHCB) e através da contribuição de especialistas.

Dados sobre o(s) ambiente(s) onde ocorrem as espécies e hábito foram baseados em observações de campo ou extraídos dos rótulos das exsicatas examinadas.

Consideraram-se ervas espécies não lenhosas, podendo estas ser terrestes, rupícolas e/ou epífitas. Espécies terrestres lenhosas, inclusive as xilopodiosas, predominantemente menores que $30 \mathrm{~cm}$, foram denominadas subarbustos; as que em geral se enquadram entre $30 \mathrm{~cm}$ e $2 \mathrm{~m}$ de altura foram denominadas arbustos; e as que geralmente são maiores que $2 \mathrm{~m}$, árvores. Espécies com hábito escandente ou rastejante, lenhosas ou não, foram enquadradas na categoria trepadeiras. E, por fim, denominaram-se parasitas aquelas hemi ou holoparasitas.

A circunscrição das famílias botânicas adotada é aquela proposta pelo APG II (APG 2003).

\section{Resultados e Discussão}

O levantamento das fanerógamas contou com 358 espécies distribuídas em 70 famílias botânicas (Tab. 1), sendo as famílias mais ricas Poaceae (43 spp.), Asteraceae (42 spp.), Fabaceae e Myrtaceae (21 spp. cada), Melastomataceae e Orchidaceae (18 spp. cada) que, juntas, correspondem a $45,5 \%$ do total de espécies levantadas.

Dentre as espécies inventariadas, 95 são arbustos, 88 ervas terrestres e 81 subarbustos. Menor é o numero de espécies arbóreas (40 spp.) e trepadeiras ou lianas (30 spp.). Oito espécies são ervas exclusivamente rupícolas, seis são ervas rupícolas ou terrestres, quatro são epífitas e duas espécies são ervas rupícolas ou epífitas (Fig. 1). 
162

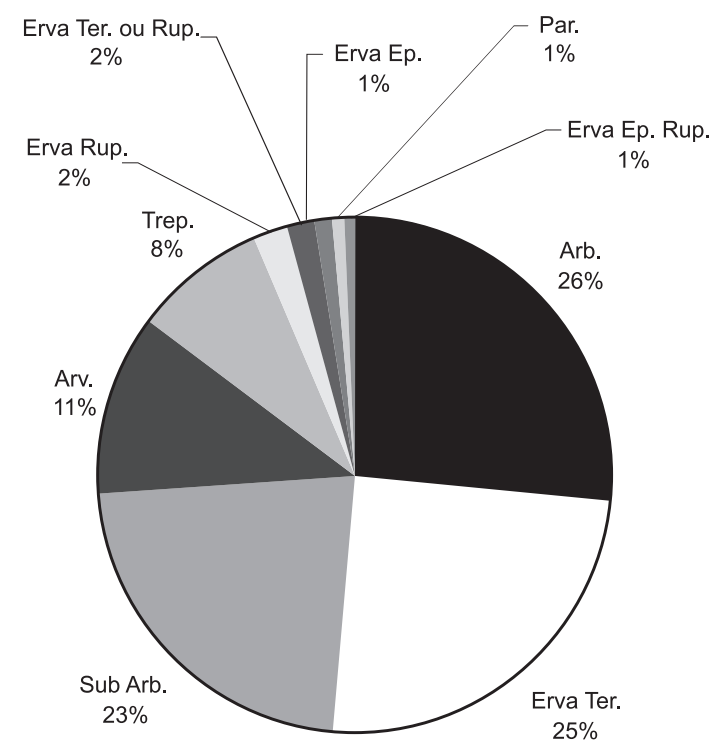

Figura 1 - Percentagem de espécies, com seus respectivos hábitos, encontradas nos campos rupestres sobre canga da Serra da Calçada, Minas Gerais, Brasil. Arb. $=$ arbusto, Arv. $=$ Árvore ou arvoreta, Erva Ep.= erva epífita, Erva Rup.= erva rupícola, Erva Ter. $=$ erva terrestre, Erva Ep. ou Rup.=erva epífita ou rupícola, Erva Ter. ou Rup. $=$ erva terrestre ou rupícola, Subarb.= subarbusto, Par. $=$ hemi ou holoparasitas, Trep. $=$ trepadeiras ou rastejantes.

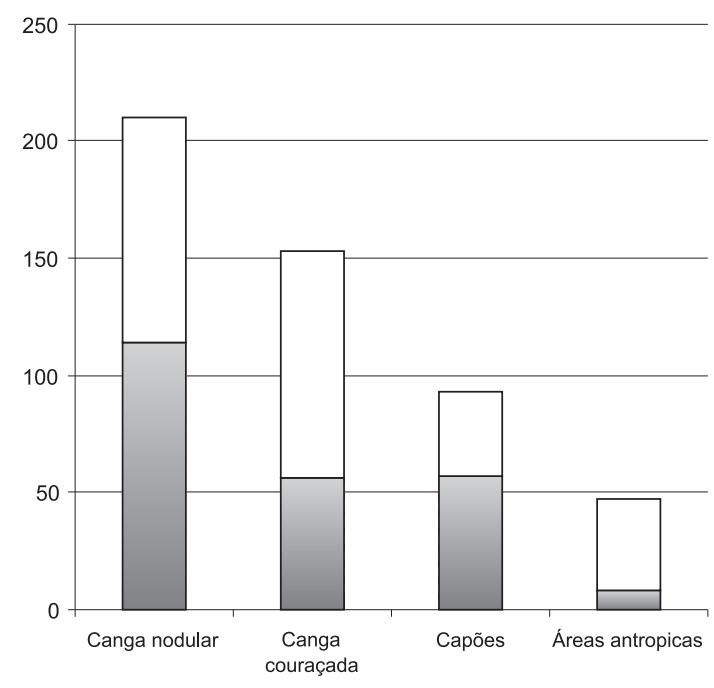

Figura 2 - Número de espécies em cada fitofisionomia dos campos rupestres sobre canga na Serra da Calçada, Minas Gerais, Brasil. As faixas coloridas representam o número de espécies exclusivas de cada fitofisionomia.
Viana, P. L. \& Lombardi, J. A.

Considerando as quatro fitofisionomias do complexo campos rupestres sobre canga, temse 210 espécies ocorrentes em campos graminosos, ou "canga nodular" sensu Rizzini (1979), 153 ocorrentes em campos rupestres sobre conglomerado hematítico ou "canga couraçada" sensu Rizinni (1979), 93 ocorrentes em pequenos capões associados aos campos rupestres sobre canga e 47 espécies encontradas em áreas antropizadas (Tab. 2).

Das 210 espécies amostradas em canga nodular, 114 , ou $54,3 \%$ foram encontradas apenas neste ambiente. Dentre as 153 espécies relacionadas à canga couraçada, 56, ou 36,6\%, são exclusivas. A maior porcentagem de espécies exclusivas a um determinado ambiente foi verificada nos capões de mata, com 61,3\% (57 das 93 spp.) de espécies que só ocorrem neste ambiente, na área amostral. Apenas oito, ou 17\%, das 47 espécies coletadas em áreas antropizadas só foram observadas nestas (Fig. 2).

Na vegetação de canga nodular, gramíneas misturadas a pequenos arbustos e subarbustos se destacam na fisionomia da vegetação atribuindo-lhe um aspecto homogêneo. Mais da metade (56\%) são ervas terrestres (Fig. 3), com destaque a várias espécies de gramíneas, como Anthaenantia lanata, Aristida recurvata, Aristida torta, Axonopus pressus, Ctenium cirrhosum, Echinolaena inflexa, Elionurus muticus, Mesosetum ferrugineum, Paspalum carinatum, Paspalum pectinatum; e Cyperaceae, como Bulbostylis paradoxa, Cyperus haspan, Rhynchospora consanguinea e $R$. terminalis; dentre os subarbustos, que correspondem a $17 \%$ das espécies levantadas neste ambiente, destacamse representantes das famílias Fabaceae e Asteraceae. São freqüentes as leguminosas Chamaecrista desvauxii, C. rotundifolia, Mimosa dolens, M. neuroloma e Stylosanthes gracilis, as compostas Aspilia foliacea, Aspilia foliosa, Aspilia subpetiolata, Baccharis multisulcata, Baccharis rufescens, Chromolaena stachyophylla, Ichthyothere integrifolia, Lessingianthus desertorum, Lessingianthus simplex, Lucilia lycopodioides, Viguiera kunthiana. Outras 
espécies subarbustivas relevantes são Annona warmingiana, Barbacenia flava, B. sellovii, Cambessedesia espora, Declieuxia oenanthoides, Galianthe thalictroides, Gomphrena arborescens, G. scapigera, Krapovickasia macrodon, Peltaea polymorpha, Pfaffia jubata, P. velutina e espécies de Croton e Mandevilla. Observamse espécies com hábito reptante ou escandente entremeadas à vegetação herbácea, como Cayaponia espelina, Galactia martii, Indigofera sp. e Zornia diphylla, e também os arbustos das famílias Asteraceae (Baccharis ramosissima, B. serrulata, Calea tomensosa,

\section{Canga couraçada}

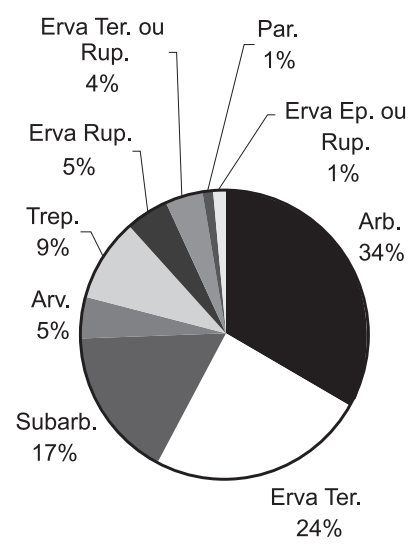

\section{Áreas antrópicas}

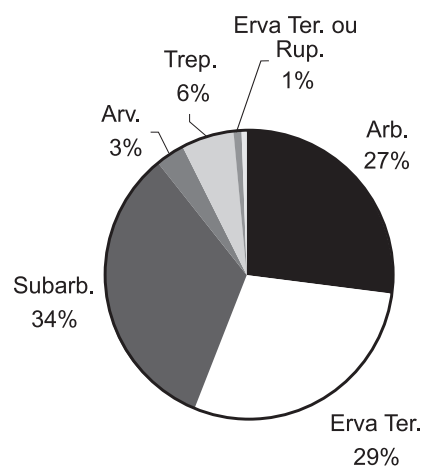

Chresta sphaerocephala, Koanophyllon adamantium, Stevia urticifolia), Malpighiaceae (Byrsonima spp. e Peixotoa tomentosa), Myrtaceae (Campomanesia spp. e Psidium spp.), Solanaceae (Solanum cladotrichum e S. isodynamum), eventualmente Vellozia compacta, e algumas espécies de Lippia. Raramente encontramse indivíduos arbóreos isolados, como Aegiphila lhotskiana, Byrsonima verbascifolia e Diospyrus sericea, que, como grande parte das espécies encontradas em canga nodular, são espécies típicas de cerrado (Mendonça et al. 1998).

\section{Canga nodular}

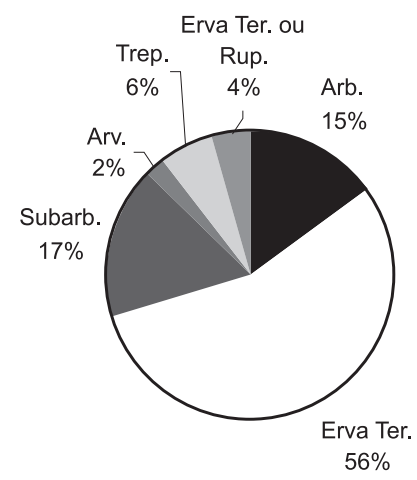

\section{Capões}

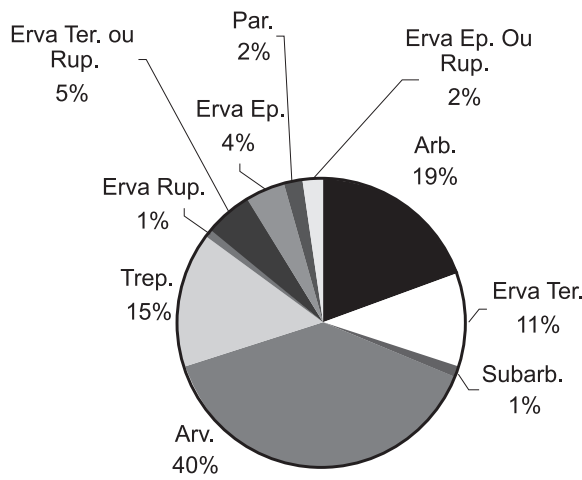

Figura 3 - Percentagem de espécies, com seus respectivos hábitos, em cada fitofisionomia dos campos rupestres sobre canga da Serra da Calçada, Minas Gerais, Brasil. Arb.= arbusto, Arv.= Árvore ou arvoreta, Erva Ep.= erva epífita, Erva Rup.= erva rupícola, Erva Ter.= erva terrestre, Erva Ep. ou Rup.=erva epífita ou rupícola, Erva Ter. ou Rup.= erva terrestre ou rupícola, Subarb.= subarbusto, Par.= hemi ou holoparasitas, Trep.= trepadeiras ou rastejantes. 
Tabela 1 - Lista das espécies fanerógamas inventariadas nos campos rupestres sobre canga da Serra da Calçada, Minas Gerais. A coluna "Am.” indica o grau de ameaça (VU: vulnerável, PE: em perigo, CR: criticamente em perigo) de acordo com Mendonça \& Lins (2000). Na coluna "Hábito", "Arb."= arbusto, "Arv."= árvore ou arvoreta, "EE"= erva epífita, "ER"= erva rupícola, "ET"= erva terrestre, "S.Arb."= subarbusto, "P"= hemi ou holoparasitas, "Tr."= trepadeiras ou rastejantes. As colunas "CC", "CN", "CM" e "AA" indicam a ocorrência em canga couraçada, canga nodular, capão de mata e/ou áreas antrópicas, respectivamente. Os "vouchers" estão representados pelos números de coleta de Pedro L. Viana (V), Julio A. Lombardi (L), João Renato Stehmann (S) e Alexandre Barros (B), ou pelo número de registro no Herbário da Universidade Federal de Minas Gerais (BHCB), caso a coleta não tenha sido realizada pelos coletores acima citados. As espécies

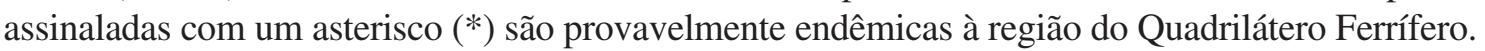

\begin{tabular}{|c|c|c|c|c|c|c|c|}
\hline Família/Espécie & Am. & Hábito & $\mathbf{C C}$ & $\mathbf{C N}$ & $\mathbf{C M}$ & $\mathbf{A A}$ & Voucher \\
\hline \multicolumn{8}{|l|}{ ACANTHACEAE } \\
\hline Justicia riparia Kameyana & & Arb. & $\mathbf{x}$ & & $\mathbf{x}$ & & V16, B25 \\
\hline Ruellia geminiflora Kunth & & S.Arb. & & $\mathbf{x}$ & & & V226, L3256, B105 \\
\hline Ruellia villosa Lindau & & Arb. & $\mathbf{x}$ & & $\mathbf{x}$ & & V7, B61, S2370, S2646 \\
\hline \multicolumn{8}{|l|}{ AMARANTHACEAE } \\
\hline Gomphrena arborescens L. f. & & S.Arb. & & $\mathbf{x}$ & & & BHCB 45368 \\
\hline Gomphrena scapigera Mart. & & S.Arb. & & $\mathbf{x}$ & & & V76 \\
\hline Gomphrena virgata Mart. & & S.Arb. & & $\mathbf{x}$ & & & V181 \\
\hline Pfaffia gnaphaloides (L. f.) Mart. & & S.Arb. & & $\mathbf{x}$ & & & V424, B100 \\
\hline Pfaffia jubata Moq. & & S.Arb. & & $\mathbf{x}$ & & & V176 \\
\hline Pfaffia velutina Moq. & & S.Arb. & & $\mathbf{x}$ & & & L3305 \\
\hline \multicolumn{8}{|l|}{ AMARYLIDACEAE } \\
\hline Habranthus irwinianus Ravenna & & ET & $\mathbf{x}$ & $\mathbf{x}$ & & & V256 \\
\hline Hippeastrum glaucescens Mart. & & ET, ER & $\mathbf{x}$ & & & & V221, L3331 \\
\hline \multicolumn{8}{|l|}{ ANACARDIACEAE } \\
\hline Tapirira obtusa (Benth.) D.J. Mitch. & & Av. & & & $\mathbf{x}$ & & V183 \\
\hline \multicolumn{8}{|l|}{ ANNONACEAE } \\
\hline Annona warmingiana Mello-Silva \& Pirani & & S.Arb. & & $\mathbf{x}$ & & & L3309 \\
\hline Guatteria sellowiana Schltdl. & VU & Av. & & & $\mathbf{x}$ & & V105, V231 \\
\hline \multicolumn{8}{|l|}{ APIACEAE } \\
\hline Eryngium eurycephalum Malme & & ET & & & $\mathbf{x}$ & $\mathbf{x}$ & V428 \\
\hline Eryngium juncifolium (Urb.) Mathias \& Constance & & ET & & & $\mathbf{x}$ & & V198, L3269, B6 \\
\hline \multicolumn{8}{|l|}{ APOCYNACEAE } \\
\hline Asclepias candida Vell. & & ET & & & $\mathbf{x}$ & & V238 \\
\hline Blepharodon nitidum (Vell.) J. F. Macbr. & & Tr. & $\mathbf{x}$ & & & & S2948 \\
\hline Ditassa aequicymosa E. Fourn. * & VU & Tr. & $\mathbf{x}$ & & $\mathbf{x}$ & & V592 \\
\hline Ditassa linearis Mart. * & VU & Tr. & $\mathbf{x}$ & & & & BHCB 76039 \\
\hline Forsteronia velloziana (A. DC.) Woodson & & Tr. & $\mathbf{x}$ & & & & B161 \\
\hline Hemipogon carassensis (Malme) Rapini & & Tr. & $\mathbf{x}$ & & & & V604 \\
\hline Mandevilla erecta (Vell.) Woodson & & S.Arb. & & $\mathbf{x}$ & & & V400 \\
\hline Mandevilla illustris (Vell.) Woodson & & S.Arb. & & $\mathbf{x}$ & & & V371, L3317 \\
\hline Mandevilla moricandiana (A. DC.) Woodson & & Tr. & & & $\mathbf{x}$ & & V536 \\
\hline Mandevilla tenuifolia (J.C. Mikan) Woodson & & S.Arb. & & $\mathbf{x}$ & & & B172 \\
\hline Matelea pedalis (E. Fourn) Fontella \& E. A. Schwarz & & Tr. & $\mathbf{x}$ & $\mathbf{x}$ & & & L3315 \\
\hline Oxypetalum appendiculatum Mart. & & Tr. & $\mathbf{x}$ & $\mathbf{x}$ & & & V538 \\
\hline Oxypetalum strictum Mart. & & Tr. & $\mathbf{x}$ & & & & S2314 \\
\hline
\end{tabular}




\section{Família/Espécie}

AQUIFOLIACEAE

Ilex cf. dumosa Reissek

Ilex sp.

\section{ARACEAE}

Anthurium minarum Sakuragui \& Mayo

Anthurium scandens (Aubl.) Engl.

\section{ASTERACEAE}

Achyrocline albicans Griseb.

Achyrocline satureioides (Lam.) DC.

Ageratum fastigiatum (Gardner) R. M. King \& H. Rob.

Aspilia foliacea Baker

Aspilia foliosa Benth. \& Hook. f.

Aspilia subpetiolata Baker

Baccharis erigeroides DC.

Baccharis multisulcata Baker

Baccharis ramosissima Gardner

Baccharis reticularia DC.

Baccharis rufescens Spreng.

Baccharis serrulata Pers.

Baccharis trimera (Less.) DC.

Barrosoa organensis (Gardner) R.M. King \& H. Rob.

Bidens alba (L.) DC.

Bidens brasiliensis Scherff

Calea tomensosa Gardner

Chaptalia integerrima (Vell.) Burkart

Chresta sphaerocephala DC.

Chromolaena stachyophylla (Spreng.) R. M. King \& H. Rob. Chrysolaena herbacea (Vell.) H. Rob.

Dasyphyllum candolleanum (Gardner) Cabrera

Eremanthus erythropappus (DC.) MacLeish

Eremanthus incanus (Less.) Less.

Hololepis pedunculata DC.

Ichthyothere integrifolia Baker

Koanophyllon adamantium (Gardner) R. M. King \& H. Rob.

Lessingianthus cephalotes (DC.) H. Rob.

Lessingianthus desertorum (Mart. ex DC.) H. Rob.

Lessingianthus simplex (Less.) H. Rob.

Lucilia lycopodioides (Less.) S. E. Freire

Lychnophora pinaster Mart.

Mikania oblongifolia DC.

Mikania officinalis Mart.

Pseudobrickellia brasiliensis (Spreng.) R.M. King \& H. Rob.

Stenocephalum tragiaefolium (DC.) Sch. Bip.

Stenophalium chionaea (DC.) Anderb.

Stevia urticifolia Thunb.

Symphyopappus brasiliensis (Gardner) R.M. King \& H. Rob.

Trixis vauthieri DC.

Viguiera kunthiana Gardner

Viguiera tenuifolia Gardner
Am. Hábito CC CN CM AA Voucher

$\begin{array}{lll}\text { Arb. } & \mathbf{x} & \text { BHCB } 41042 \\ \text { Arb. } & \mathbf{x} & \text { BHCB } 49532\end{array}$

$\begin{array}{llll}\text { ET } & \mathbf{x} & \mathbf{x} & \text { V2, S2648 } \\ \text { EE } & & \mathbf{x} & \text { V696 }\end{array}$

$\begin{array}{lll}\text { Arb. } & \mathbf{x} & \text { V138 }\end{array}$

$\begin{array}{llll}\text { Arb. } & \mathbf{x} & \mathbf{x} & \text { V135 }\end{array}$

$\begin{array}{lllll}\text { Arb. } & \mathbf{x} & \mathbf{x} & \mathbf{x} & \text { V17 }\end{array}$

$\begin{array}{lll}\text { S.Arb. } & \mathbf{x} & \text { V166, V223 }\end{array}$

$\begin{array}{lll}\text { S.Arb. } & \mathbf{x} & \text { V318 }\end{array}$

$\begin{array}{lll}\text { S.Arb. } & \mathbf{x} & \text { V30 }\end{array}$

Arb. $\quad \mathbf{x} \quad \mathbf{x} \quad$ L3289

S.Arb. $\quad \mathbf{x} \quad$ V165

$\begin{array}{llll}\text { Arb. } & \mathbf{x} & \mathbf{x} & \text { V18 }\end{array}$

Arb. $\quad \mathbf{x} \quad \mathbf{x} \quad$ V66, S2412

$\begin{array}{llll}\text { S.Arb. } & \mathbf{x} & \mathbf{x} & \text { V190 }\end{array}$

Arb. $\quad \mathbf{x}$

S.Arb. $\quad \mathbf{x}$

Arb. $\quad \mathbf{x}$

S.Arb. $\quad \mathbf{x}$

Tr.

x V97, B135, L3262

x L3951

S2398, 2322

x B127

x $\quad$ V625

S2389, B102

x $\mathbf{L} 3286$

S2638

L3299

B113

V5, S2635

V6

V31

V791

V175

V799

BHCB 49563

L3259

B177

B4

V4, 30

L3330

L3293

V222

BHCB 49560

B33

V621

V429

V63

V247

BHCB 49571 


\section{Família/Espécie}

BIGNONIACEAE

Jacaranda caroba (Vell.) A. DC.

Pyrostegia venusta (Ker Gawl.) Miers

Anemopaegma arvense (Vell.) Stellfeld ex de Souza

BORAGINACEAE

Cordia curassavica (Jacq.) Roem. \& Schult.

BROMELIACEAE

Aechmea bromeliifolia (Rudge) Baker

Billbergia elegans Mart. ex Schult. \& Schult. f.

Dyckia consimilis $\mathrm{Mez} *$

Tillandsia recurvata (L.) L.

Vriesea minarum L.B. Sm. *

\section{Am. Hábito CC CN CM AA Voucher}

$\begin{array}{llllll}\text { Arb. } & & \text { x } & & \text { x } & \text { V140, L3254 } \\ \text { Tr. } & \text { x } & \text { x } & \text { x } & \text { x } & \text { V623 } \\ \text { Tr. } & & & \text { x } & & \text { BHCB 49555 }\end{array}$

$\begin{array}{llllll}\text { Arb. } & \mathbf{x} & \mathbf{x} & & \mathbf{x} & \text { V537 } \\ & & & & \\ \text { ER, EE } & \mathbf{x} & & \mathbf{x} & & \text { V118 } \\ \text { EE } & & & \mathbf{x} & \text { V351 } \\ \text { ER } & \mathbf{x} & & & \text { V374 } \\ \text { EE } & & & \mathbf{x} & \text { V71 } \\ \text { ER } & \mathbf{x} & & & \text { V850 }\end{array}$

V370

Arthrocereus glaziovii (Schumann.) N. P. Taylor \& D. C. Zappi * CR ER x

Av.

B69, 159, 137, V214

Maytenus gonoclada Mart.

Av.

Clusia arrudae Planch. \& Triana ex Engl.

Kielmeyera variabilis Mart. \& Zucc.

Vismia brasiliensis Choisy

$\begin{array}{llll}\text { Av. } & & \mathbf{x} & \text { V10, S2402, S2403 } \\ \text { S.Arb. } & \mathbf{x} & & \text { V227, S2649 } \\ \text { Av. } & & \mathbf{x} & \text { L3952 } \\ & & & \\ & & & \text { V407 } \\ \text { ET } & & \mathbf{x} & \text { V406 } \\ \text { ET } & & \mathbf{x} & \end{array}$

Commelina erecta $\mathrm{L}$

Dichorisandra hexandra (Aubl.) Standl.

CONVOLVULACEAE

Evolvulus filipes Mart.

Evolvulus macroblepharis Mart.

Ipomoea polymorpha Riedel

Jacquemontia linarioides Meisn.

ET $\quad \mathbf{x} \quad \mathbf{x}$

ET

Tr.

S.Arb.

x $\quad$ V406

CUCURBITACEAE

Cayaponia espelina (Silva Manso) Cogn.

CUNONIACEAE

Lamanonia ternata Vell.

Av.

V242

\section{CYPERACEAE}

Bulbostylis fimbriata (Nees) C. B. Clarke

Bulbostylis paradoxa (Spreng.) Lindm.

Cyperus aggregatus (Willd.) Endl.

Cyperus haspan L.

Cyperus subcastaneus D. A. Simpson

Rhynchospora consanguinea (Kunth) Boeck.

Rhynchospora exaltata Kunth

Rhynchospora terminalis Nees ex Steud.

Rhynchospora sp.

Trilepis lhotzkiana Nees ex Arn.

\section{DIOSCOREACEAE}

Dioscorea debilis Uline

$\begin{array}{lllll}\text { ET } & \mathbf{x} & \mathbf{x} & & \text { V582 } \\ \text { ET } & & \mathbf{x} & & \text { V204, S2304 } \\ \text { ET } & \mathbf{x} & \mathbf{x} & \mathbf{x} & \text { V423, 583 } \\ \text { ET } & & \mathbf{x} & \mathbf{x} & \text { V630 } \\ \text { ET } & \mathbf{x} & & & \text { BHCB 79734 } \\ \text { ET } & & \mathbf{x} & & \text { V260, B104 } \\ \text { ET } & \mathbf{x} & \mathbf{x} & & \text { V81 } \\ \text { ET } & & \mathbf{x} & & \text { V197 } \\ \text { ET } & & \mathbf{x} & & \text { BHCB 79732 } \\ \text { ET } & \mathbf{x} & & & \text { V798 }\end{array}$

V549, S2313,

x $\mathbf{S} 2324$

V347

V357, S2647

V962

x $\quad$ BHCB 80471 


\section{Família/Espécie}

EBENACEAE

Diospyrus sericea A. DC.

ERICACEAE

Agarista eucalyptoides G. Don.

Gaylussacia brasiliensis Meissn.

Gaylussacia chamissonis Meissn.

\section{ERIOCAULACEAE}

Paepalanthus blepharocnemis Mart. ex Körn.

\section{ERYTHROXYLACEAE}

Erythroxylum bicolor O. E. Schulz

Erythroxylum suberosum A.St.-Hil.

Erythroxylum sp.

\section{EUPHORBIACEAE}

Alchornea triplinervia (Spreng.) Müll.Arg.

Chamaesyce potentilloides (Boiss.) Criozat

Croton antisiphiliticus Mart.

Croton atrorufus Müll.Arg.

Croton fuscescens Spreng.

Croton migrans Casar

Croton sp1.

Croton sp2.

Sapium haematospermum Müll.Arg.

Sebastiania glandulosa (Sw.) Müll.Arg.

Indeterminada

\section{FABACEAE}

Bauhinia rufa (Bong.) Steud.

Chamaecrista desvauxii (Collad.) Killip

Chamaecrista rotundifolia (Pers.) Greene

Chamaecrista secunda (Benth.) H.S. Irwin \& Barneby

Copaifera langsdorffii Desf.

Galactia martii DC.

Indigofera sp.

Mimosa calodendron Mart. ex Benth.*

Mimosa dolens Vell.

Mimosa neuroloma Benth.

Mimosa pogocephala Benth.

Mimosa setistipula Benth.

Periandra mediterranea (Vell.) Taub.

Senna rugosa (G. Don) H. S. Irwin \& Barneby

Stylosanthes gracilis Kunth

Zornia diphylla (L.) Pers.

Indeterminada 1

Indeterminada 2

Indeterminada 3

Indeterminada 4

Indeterminada 5

\section{GESNERIACEAE}

Paliavana sericiflora Benth.

Sinningia allagophylla (Mart.) Wiehler

Sinningia rupicola (Mart.) Wiehler*

\section{Am. Hábito CC CN CM AA Voucher}

Av.

Av.

$\mathbf{x}$

S.Arb.

ET

Arb.

Arb.

Arb.

Av

ET

S.Arb.

S.Arb.

Arb.

Arb. $\quad \mathbf{x}$

Arb. $\quad \mathbf{x}$

Arb.

Arb. $\quad \mathbf{x} \quad \mathbf{x}$

Arb. $\quad \mathbf{x}$

S.Arb.

Arb. $\quad \mathbf{x}$

S.Arb. $\quad \mathbf{x}$

S.Arb. $\quad \mathbf{x} \quad \mathbf{x}$

S.Arb. $\quad \mathbf{x}$

Av.

Tr.

Tr.

Arb. $\quad \mathbf{x}$

S.Arb. $\quad \mathbf{x}$

S.Arb. $\quad \mathbf{x} \quad \mathbf{x}$

Arb.

S.Arb. $\quad \mathbf{x} \quad \mathbf{x}$

Arb. $\quad \mathbf{x} \quad \mathbf{x}$

Arb. $\quad \mathbf{x} \quad \mathbf{x}$

S.Arb.

Tr.

S.Arb.

ET

Tr.

Tr.

ET $\mathbf{x}$

$\mathbf{x}$

$\mathbf{x}$

$\mathbf{x}$

$\mathbf{x}$

$\mathbf{x}$

V194

V233

BHCB 80405

V117

BHCB 49570

BHCB 49587
V811

V177

V246

B129

V237, L3281

BHCB 41037

BHCB 80469

BHCB 75505

V376

V61,V100,V358,B31,S2401

BHCB 69931

V94

V410

V425

V235, B114, B150

V113

V116, V427

x BHCB 69921

V544

BHCB 49517

V375, V597

S2415

V96

V409

BHCB 40797

V174

x BHCB 80380, 80381, 80383

BHCB 49581

BHCB 76036

BHCB 40622

BHCB 40646

x $\quad$ BHCB 49519 


\section{Família/Espécie}

IRIDACEAE

Neomarica rupestris (Ravenna) N.S. Chukr

Pseudotrimezia sp.

Sisyrinchium luzula Klotzsch

Sisyrinchium vaginatum Spreng.

\section{LAMIACEAE}

Aegiphila lhotskiana Cham.

Eriope macrostachya Mart. ex Benth.

Eriope sp.

Hyptis lippioides Pohl ex Benth.

Salvia sp.

Vitex cymosa Bertero ex Spreng.

Vitex polygama Cham.

Indeterminada

\section{LAURACEAE}

Cinnamomum quadrangulum (Meisn.) Kosterm.

Ocotea pulchella (Nees) Mez

Ocotea tristis (Nees \& Mart.) Mez

\section{LOGANIACEAE}

Spigelia schlechtendaliana Mart.

\section{LORANTHACEAE}

Struthanthus flexicaulis Mart.

Tripodanthus acutifolius (Ruiz \& Pav.) Tiegh.

\section{LYTHRACEAE}

Cuphea ericoides Cham. \& Schltdl.

Cuphea thymoides Cham. \& Schltd.

Diplusodon cf. hirsutus (Cham. \& Schltdl.) DC.

Diplusodon myrsinitis DC.

Lafoensia pacari A.St. Hil

\section{MALPIGHIACEAE}

Banisteriopsis campestris (A. Juss.) Little Banisteriopsis malifolia (Nees \& Mart.) B. Gates Byrsonima sericea DC.

Byrsonima subterranea Brade \& Markgr.

Byrsonima variabilis A. Juss.

Byrsonima verbascifolia (L.) DC.

Camarea hirsuta A.St.-Hil.

Heteropterys umbellata A. Juss.

Peixotoa tomentosa A. Juss.

Tetrapterys microphylla (A. Juss.) Nied.

\section{MALVACEAE}

Krapovickasia macrodon (DC.) Fryxell

Peltaea polymorpha (A. St.-Hil.) Krapov. \& Cristóbal Sida glaziovii K. Schum.

Waltheria indica $\mathrm{L}$.

\section{MELASTOMATACEAE}

Cambessedesia corymbosa DC.

Cambessedesia espora DC.
Am. Hábito CC CN CM AA Voucher

$\begin{array}{lllll}\text { ET } & & & \mathbf{x} & \text { V420 } \\ \text { ET } & & \mathbf{x} & & \text { V220 } \\ \text { ET } & & \mathbf{x} & & \text { V187 } \\ \text { ET } & \mathbf{x} & \mathbf{x} & & \text { V92, V225, L3229 }\end{array}$

\begin{tabular}{|c|c|c|c|c|}
\hline Av. & & $\mathbf{x}$ & $\mathbf{x}$ & V130, V372 \\
\hline Arb. & $\mathbf{x}$ & $\mathbf{x}$ & & V9, B63 \\
\hline Arb. & & $\mathbf{x}$ & & V167, B107 \\
\hline Arb. & $\mathbf{x}$ & $\mathbf{x}$ & & L3255, B52 \\
\hline S.Arb. & & $\mathbf{x}$ & & ВНCB 49554, 40648 \\
\hline Av. & $\mathbf{x}$ & & & L3292 \\
\hline Av. & & & $\mathbf{x}$ & V348 \\
\hline Arb. & & $\mathbf{x}$ & & ВНСВ 80474 \\
\hline
\end{tabular}

$\begin{array}{lllllll}\text { VU } & \text { Arb. } & \mathbf{x} & \mathbf{x} & & & \text { V103, V416 } \\ \text { PE } & \text { Av. } & & & \mathbf{x} & \text { B139 } \\ & \text { Arb. } & \mathbf{x} & & & & \text { V581, B38 }\end{array}$

$\begin{array}{llll}\text { S.Arb. } & \mathbf{x} & \mathbf{x} & \text { V546, L3264 }\end{array}$

$\begin{array}{llllll}\mathrm{P} & \mathbf{x} & \mathbf{x} & \mathbf{x} & \mathrm{V} 193\end{array}$

$\begin{array}{lll}\mathrm{P} & \mathbf{x} & \text { V531, L3953 }\end{array}$

$\begin{array}{llllll}\text { S.Arb. } & \mathbf{x} & \mathbf{x} & & & \text { V180 } \\ \text { S.Arb. } & \mathbf{x} & \mathbf{x} & & \mathbf{x} & \text { V356 } \\ \text { Arb. } & \mathbf{x} & \mathbf{x} & & & \text { V603 } \\ \text { Arb. } & \mathbf{x} & \mathbf{x} & & & \text { B1 } \\ \text { Av. } & & & \mathbf{x} & & \text { V54 }\end{array}$

$\begin{array}{lll}\text { Tr. } & \mathbf{x} & \text { V349, } 401\end{array}$

$\begin{array}{llll}\text { Tr. } & \mathbf{x} & \mathbf{x} & \text { V211 }\end{array}$

Arb. $\quad$ x $\quad$ BHCB 69920, 75617

$\begin{array}{lll}\text { S.Arb. } & \mathbf{x} & \text { L3306 }\end{array}$

$\begin{array}{lll}\text { Arb. } & \mathbf{x} & \text { B116 }\end{array}$

$\begin{array}{lll}\text { Arb. } & \mathbf{x} & \text { V159, L3274 }\end{array}$

V210

L3291

V40

V110

\begin{tabular}{|c|c|c|c|}
\hline S.Arb. & $\mathbf{x}$ & & V354 \\
\hline S.Arb. & $\mathbf{x}$ & & V380 \\
\hline S.Arb. & & $\mathbf{x}$ & V600 \\
\hline S.Arb. & & $\mathbf{x}$ & V192, V598 \\
\hline S.Arb. $\quad \mathbf{x}$ & $\mathbf{x}$ & & V433, B183 \\
\hline S.Arb. & $\mathbf{x}$ & & V23 \\
\hline
\end{tabular}




\begin{tabular}{|c|c|c|c|c|c|c|c|}
\hline Família/Espécie & Am. & Hábito & $\mathbf{C C}$ & $\mathbf{C N}$ & $\mathbf{C M}$ & $\mathbf{A A}$ & Voucher \\
\hline Cambessedesia hilariana (Kunth) DC. & & S.Arb. & $\mathbf{x}$ & $\mathbf{x}$ & & & V584 \\
\hline Lavoisiera cf. imbricata DC. & & Arb. & $\mathbf{x}$ & & & & BHCB 49515 \\
\hline Leandra aff. aurea (Cham.) Cogn. & & Av. & $\mathbf{x}$ & & $\mathbf{x}$ & & BHCB 41046 \\
\hline Leandra australis (Cham.) Cogn. & & Arb. & $\mathbf{x}$ & & & & BHCB 40838 \\
\hline Leandra aff. cancellata Cogn. & & Arb. & $\mathbf{x}$ & & $\mathbf{x}$ & & BHCB 49524 \\
\hline Leandra sp1. & & Arb. & & & $\mathbf{x}$ & & BHCB 40662 \\
\hline Leandra $\mathrm{sp} 2$. & & Arb. & & & $\mathbf{x}$ & & BHCB 45364 \\
\hline Miconia chartacea Triana & & Av. & & & $\mathbf{x}$ & & BHCB 73729 \\
\hline Miconia pepericarpa Mart. ex DC. & & Arb. & $\mathbf{x}$ & & $\mathbf{x}$ & & V350, V596 \\
\hline Miconia sellowiana Naudin & & Av. & & & $\mathbf{x}$ & & V119, V182 \\
\hline Miconia sp. & & Av. & & & $\mathbf{x}$ & & BHCB 69918 \\
\hline Microlicia pseudoscoparia Cogn. & & S.Arb. & $\mathbf{x}$ & & & & ВНCB 69919 \\
\hline Tibouchina herbacea (DC.) Cogn. & & S.Arb. & & $\mathbf{x}$ & & & V397 \\
\hline Tibouchina hieracioides (DC.) Cogn. & & S.Arb. & & $\mathbf{x}$ & & & BHCB 80475 \\
\hline Tibouchina multiflora (Gardn.) Cogn. & & Arb. & $\mathbf{x}$ & $\mathbf{x}$ & & & V15 \\
\hline Trembleya parviflora (Don.) Cogn. & & Arb. & $\mathbf{x}$ & & & & V95 \\
\hline \multicolumn{8}{|l|}{ MELIACEAE } \\
\hline Cabralea canjerana (Vell.) Mart. & & Av. & & & $\mathbf{x}$ & & V111, V124 \\
\hline \multicolumn{8}{|l|}{ MENISPERMACEAE } \\
\hline Cissampelos ovalifolia DC. & & S.Arb. & & $\mathbf{x}$ & & $\mathbf{x}$ & B165, B171, L3294 \\
\hline \multicolumn{8}{|l|}{ MORACEAE } \\
\hline Dorstenia brasiliensis Lam. & & ET & $\mathbf{x}$ & & & & S2328, L3338 \\
\hline \multicolumn{8}{|l|}{ MYRSINACEAE } \\
\hline Myrsine guianensis (Aubl.) Kuntze & & Av. & & $\mathbf{x}$ & & & L3288 \\
\hline Myrsine lancifolia Mart. & & Arb. & $\mathbf{x}$ & & & & S2408, B157 \\
\hline Myrsine umbellata Mart. & & Av. & & & $\mathbf{x}$ & & V243 \\
\hline \multicolumn{8}{|l|}{ MYRTACEAE } \\
\hline Blepharocalyx salicifolius (Kunth) O. Berg & & Arb. & & $\mathbf{x}$ & $\mathbf{x}$ & & V359, V384, V685 \\
\hline Campomanesia adamantium (Cambess.) O. Berg & & Arb. & & $\mathbf{x}$ & & & V184, B09 \\
\hline Campomanesia pubescens (DC.) O. Berg & & S.Arb. & & $\mathbf{x}$ & & & V230, V353 \\
\hline Campomanesia rufa (O. Berg) Nied. & & S.Arb. & & $\mathbf{x}$ & & & V373 \\
\hline Campomanesia sessiliflora (O. Berg) Mattos & & Arb. & & $\mathbf{x}$ & & & L3249 \\
\hline Campomanesia sp. & & S.Arb. & & $\mathbf{x}$ & & & BHCB 71656 \\
\hline Eugenia bimarginata $\mathrm{O}$. Berg & & S.Arb. & $\mathbf{x}$ & $\mathbf{x}$ & & & B21 \\
\hline Eugenia cf. eurysepala Kiaersk. & & Arb. & & & $\mathbf{x}$ & & BHCB 52077 \\
\hline Eugenia punicifolia (Kunth) DC. & & Arb. & $\mathbf{x}$ & $\mathbf{x}$ & & & V229, S2640 \\
\hline Eugenia sonderiana $\mathrm{O}$. Berg & & Arb. & & & $\mathbf{x}$ & & V362 \\
\hline Gomidesia kunthiana O. Berg & & Arb. & & & $\mathbf{x}$ & & V548 \\
\hline Myrceugenia alpigena (DC.) Landrum & & Av. & & & $\mathbf{x}$ & & V545, V601 \\
\hline Myrcia crassifolia (Miq.) Kiaersk. & & Av. & $\mathbf{x}$ & & $\mathbf{x}$ & & V119, V377 \\
\hline Myrcia lasiantha DC. & & Av. & & & $\mathbf{x}$ & & V93 \\
\hline Myrcia splendens (Sw.) DC. & & Av. & $\mathbf{x}$ & & $\mathbf{x}$ & & V185, V382 \\
\hline Myrciaria glanduliflora (Kiaersk.) Mattos \& D. Legrand & & Av. & & & $\mathbf{x}$ & & L3276 \\
\hline Psidium cinereum Mart. ex DC. & & Arb. & & $\mathbf{x}$ & & & V129, BHCB 69908 \\
\hline Psidium luridum (Spreng.) Burret & & Arb. & & $\mathbf{x}$ & & & B128 \\
\hline Psidium obovatum Mart. ex DC. & & Arb. & & $\mathbf{x}$ & & & BHCB 71651 \\
\hline Siphoneugena densiflora O. Berg & & Av. & $\mathbf{x}$ & & $\mathbf{x}$ & & V109, V761 \\
\hline Psidium firmum $\mathrm{O}$. Berg & & Arb. & & $\mathbf{x}$ & & & BHCB 49531 \\
\hline \multicolumn{8}{|l|}{ NYCTAGINACEAE } \\
\hline Guapira obtusata (Jacq.) Little & & Av. & & & $\mathbf{x}$ & & V107 \\
\hline
\end{tabular}




\begin{tabular}{|c|c|c|c|c|c|c|}
\hline Família/Espécie & Hábito & $\mathbf{C C}$ & $\mathbf{C N}$ & $\mathbf{C M}$ & $\mathbf{A A}$ & Voucher \\
\hline \multicolumn{7}{|l|}{ OCHNACEAE } \\
\hline Ouratea floribunda Engl. & Arb. & & $\mathbf{x}$ & & & V200 \\
\hline Ouratea semiserrata (Mart. \& Nees) Engl. & Av. & & & $\mathbf{x}$ & & V13 \\
\hline \multicolumn{7}{|l|}{ ORCHIDACEAE } \\
\hline Acianthera limae (Porto \& Brade) Pridgeon \& M.W. Chase & ET, ER & $\mathbf{x}$ & & $\mathbf{x}$ & & V707 \\
\hline Acianthera teres (Lindl.) Luer & ER & $\mathbf{x}$ & & & & V550 \\
\hline Bifrenaria tyrianthina (Loudon) Rchb. f. & ET, ER & $\mathbf{x}$ & & $\mathbf{x}$ & & V132 \\
\hline Epidendrum campestre Lindl. & ER & $\mathbf{x}$ & & & & V60 \\
\hline Epidendrum martianum Lindl. & ET & $\mathbf{x}$ & & & & V127 \\
\hline Epidendrum secundum Jacq. & ET, ER & $\mathbf{x}$ & $\mathbf{x}$ & $\mathbf{x}$ & $\mathbf{x}$ & V70 \\
\hline Galeandra montana Barb. Rodr. & ET & $\mathbf{x}$ & $\mathbf{x}$ & $\mathbf{x}$ & & V486 \\
\hline Habenaria josephensis Barb. Rodr. & ET & & $\mathbf{x}$ & & & ВНCB 69912 \\
\hline Habenaria sp. & ET & & $\mathbf{x}$ & & & BHCB 80437 \\
\hline Oncidium gracile Lindl.* & ER & $\mathbf{x}$ & & & & V624 \\
\hline Oncidium pirarene Rchb. f. & ET, ER & $\mathbf{x}$ & $\mathbf{x}$ & $\mathbf{x}$ & $\mathbf{x}$ & V578 \\
\hline Oncidium warmingii Reichb. f. & $\mathrm{ER}, \mathrm{EE}$ & $\mathbf{x}$ & & $\mathbf{x}$ & & V533 \\
\hline Prescottia montana Rodrig. & ET & & & $\mathbf{x}$ & & V102 \\
\hline Prescottia oligantha Lindl. & ET & & & $\mathbf{x}$ & & V126 \\
\hline Sacoila lanceolata (Aubl.) Garay & ET & $\mathbf{x}$ & $\mathbf{x}$ & $\mathbf{x}$ & $\mathbf{x}$ & V244 \\
\hline Sophronitis caulescens (Lindl.) C. Berg \& M.W. Chase & ER & $\mathbf{x}$ & & & & V62 \\
\hline Sophronitis crispata (Thunb.) C. Berg \& M.W. Chase & $\mathrm{ER}$ & $\mathbf{x}$ & & $\mathbf{x}$ & & V771 \\
\hline Zygopetalum mackayi Hook. & $\mathrm{ET}, \mathrm{ER}$ & $\mathbf{x}$ & & $\mathbf{x}$ & & V638 \\
\hline \multicolumn{7}{|l|}{ OROBACHACEAE } \\
\hline Buchnera lavandulacea Cham. \& Schlecht. & S.Arb. & & $\mathbf{x}$ & & & $\mathrm{B} 8$ \\
\hline \multicolumn{7}{|l|}{ PASSIFLORACEAE } \\
\hline Passiflora amethystina J. C. Mikan & Tr. & & & $\mathbf{x}$ & & V599 \\
\hline Passiflora haematostigma Mart. ex Mast. & $\operatorname{Tr}$ & $\mathbf{x}$ & & & & V772 \\
\hline \multicolumn{7}{|l|}{ PHYLLANTHACEAE } \\
\hline Phyllanthus rosellus Müll. Arg. & ET & $\mathbf{x}$ & $\mathbf{x}$ & & $\mathbf{x}$ & S2400 \\
\hline \multicolumn{7}{|l|}{ PHYTOLACACEAE } \\
\hline Microtea paniculata Moq. & ET & $\mathbf{x}$ & & & $\mathbf{x}$ & V574 \\
\hline \multicolumn{7}{|l|}{ PIPERACEAE } \\
\hline Peperomia decora Dahlst. & $\mathrm{ER}$ & $\mathbf{x}$ & & & & V12, V355 \\
\hline Peperomia galioides Kunth & $\mathrm{ET}$ & $\mathbf{x}$ & & & & V403 \\
\hline \multicolumn{7}{|l|}{ POACEAE } \\
\hline Andropogon bicornis L. & ET & $\mathbf{x}$ & $\mathbf{x}$ & & $\mathbf{x}$ & V631 \\
\hline Andropogon gayanus Kunth & ET & & & & $\mathbf{x}$ & V634 \\
\hline Andropogon ingratus Hack. & ET & $\mathbf{x}$ & & & & V575 \\
\hline Andropogon leucostachyus Kunth & ET & $\mathbf{x}$ & $\mathbf{x}$ & & $\mathbf{x}$ & V381 \\
\hline Anthaenantia lanata (Kunth) Benth. & ET & & $\mathbf{x}$ & & & V257 \\
\hline Aristida flaccida Trin. \& Rupr. & ET & & $\mathbf{x}$ & & & V796 \\
\hline Aristida recurvata Kunth & ET & & $\mathbf{x}$ & & & V645 \\
\hline Aristida torta (Nees) Kunth & ET & & $\mathbf{x}$ & & & V574, V646 \\
\hline Axonopus fissifolius (Raddi) Kuhlm. & ET & & $\mathbf{x}$ & & $\mathbf{x}$ & V964 \\
\hline Axonopus marginatus (Trin.) Chase & ET & & $\mathbf{x}$ & & & V368 \\
\hline Axonopus pressus (Nees ex Steud.) Parodi & ET & & $\mathbf{x}$ & & & V965 \\
\hline Axonopus siccus Kuhlm. & ET & $\mathbf{x}$ & $\mathbf{x}$ & & & V629 \\
\hline Calamagrostis viridiflavescens (Poir.) Steud. & ET & & $\mathbf{x}$ & & & V974 \\
\hline Chloris pycnothrix Trin. & $\mathrm{ET}$ & & & & $\mathbf{x}$ & V684 \\
\hline
\end{tabular}




\begin{tabular}{|c|c|c|c|c|c|c|}
\hline Família/Espécie & Hábito & $\mathbf{C C}$ & $\mathbf{C N}$ & $\mathbf{C M}$ & AA & Voucher \\
\hline Ctenium cirrhosum (Nees) Kunth & ET & & $\mathbf{x}$ & & & V426 \\
\hline Echinolaena inflexa (Poir.) Chase & ET & $\mathbf{x}$ & $\mathbf{x}$ & & & V415 \\
\hline Elionurus muticus (Spreng.) Kuntze & ET & & $\mathbf{x}$ & & & V206 \\
\hline Eragrostis curvula (Schrad.) Nees & ET & & $\mathbf{x}$ & & & V794 \\
\hline Eragrostis polytricha Nees & ET & & $\mathbf{x}$ & & & V367 \\
\hline Eriochrysis holcoides (Nees) Kuhlm. & ET & & $\mathbf{x}$ & & & V973 \\
\hline Hyparrhenia rufa (Nees) Stapf & ET & & & & $\mathbf{x}$ & V637 \\
\hline Megathyrsus maximus (Jacq.) B.K. Simon \& S.W.L. Jacobs & ET & & & & $\mathbf{x}$ & V636 \\
\hline Melinis minutiflora $\mathrm{P}$. Beauv. & ET & $\mathbf{x}$ & $\mathbf{x}$ & & $\mathbf{x}$ & V90 \\
\hline Melinis repens (Willd.) C. E. Hubb. & ET & $\mathbf{x}$ & $\mathbf{x}$ & & $\mathbf{x}$ & V633 \\
\hline Mesosetum ferrugineum (Trin.) Chase & ET & & $\mathbf{x}$ & & & V363 \\
\hline Mesosetum loliiforme (Hochst. ex Steud.) Chase & ET & & $\mathbf{x}$ & & & V975 \\
\hline Microchloa indica (L. f.) P. Beauv. & ET & $\mathbf{x}$ & & & $\mathbf{x}$ & V590 \\
\hline Panicum cyanescens Nees & ET & $\mathbf{x}$ & $\mathbf{x}$ & & & V653 \\
\hline Panicum pseudisachne $\mathrm{Mez}$ & ET & $\mathbf{x}$ & $\mathbf{x}$ & & $\mathbf{x}$ & V596, V594 \\
\hline Panicum rude Nees & ET & & & & $\mathbf{x}$ & V414 \\
\hline Panicum sellowii Nees & ET & $\mathbf{x}$ & & & $\mathbf{x}$ & V627 \\
\hline Paspalum carinatum Humb. \& Bonpl. ex Flüggé & ET & & $\mathbf{x}$ & & & V968 \\
\hline Paspalum maculosum Trin. & ET & & $\mathbf{x}$ & & & V969 \\
\hline Paspalum pectinatum Nees & ET & & $\mathbf{x}$ & & & V364 \\
\hline Paspalum polyphyllum Nees & ET & $\mathbf{x}$ & $\mathbf{x}$ & & $\mathbf{x}$ & V628 \\
\hline Paspalum scalare Trin. & ET & $\mathbf{x}$ & & & & V589 \\
\hline Setaria parviflora (Poir.) Kerguélen & ET & $\mathbf{x}$ & $\mathbf{x}$ & & $\mathbf{x}$ & V967 \\
\hline Sporobolus metallicolus Longhi Wagner \& Boechat & ET & $\mathbf{x}$ & & & & V580 \\
\hline Sporobolus reflexus Boechat \& Longhi Wagner & ET & & $\mathbf{x}$ & & & V217, V365 \\
\hline Sporolobus acuminatus (Trin.) Hack. & ET & & $\mathbf{x}$ & & & V971 \\
\hline Thrasyopsis repanda (Nees) Parodi & ET & & $\mathbf{x}$ & & & V216 \\
\hline Trachypogon spicatus (L. f.) Kuntze & ET & & $\mathbf{x}$ & & & V660 \\
\hline Tristachya leiostachya Nees & ET & & $\mathbf{x}$ & & & V133 \\
\hline \multicolumn{7}{|l|}{ POLYGALACEAE } \\
\hline Monnina stenophylla A. St. Hil \& Moq. & S.Arb. & & $\mathbf{x}$ & & & L3311 \\
\hline Polygala hirsuta A. St.-Hil. \& Moq. & ET & & $\mathbf{x}$ & & & BHCB 49572 \\
\hline Polygala paniculata $\mathrm{L}$. & ET & & & & $\mathbf{x}$ & V422 \\
\hline \multicolumn{7}{|l|}{ POLYGONACEAE } \\
\hline Coccoloba scandens Casar. & Tr. & $\mathbf{x}$ & & $\mathbf{x}$ & & V522 \\
\hline \multicolumn{7}{|l|}{ ROSACEAE } \\
\hline Prunus myrtifolia (L.) Urb. & Av. & & & $\mathbf{x}$ & & V539 \\
\hline \multicolumn{7}{|l|}{ RUBIACEAE } \\
\hline Alibertia vaccinioides K. Schum. & Arb. & $\mathbf{x}$ & & & & BHCB 75522,75523 \\
\hline Coccocypselum lanceolatum (Ruiz \& Pav.) Pers. & ET & & & $\mathbf{x}$ & & V213, V430 \\
\hline Declieuxia oenanthoides Mart. \& Zucc. ex Schult. \& Schult. f. & S.Arb. & $\mathbf{x}$ & $\mathbf{x}$ & & & V319 \\
\hline Diodia sp. & Erva & $\mathbf{x}$ & & & & BHCB 76035 \\
\hline Faramea cyanea Müll. Arg. & Av. & & & $\mathbf{x}$ & & V378, V607 \\
\hline Galianthe cf. angustifolia (Cham. \& Schltdl.) E.L. Cabral & S.Arb. & $\mathbf{x}$ & $\mathbf{x}$ & & & V417 \\
\hline Galianthe thalictroides (K. Schum.) E.L. Cabral & S.Arb. & & $\mathbf{x}$ & & & V14, V418 \\
\hline Mitracarpus sp. & S.Arb. & & $\mathbf{x}$ & & $\mathbf{x}$ & BHCB 80473 \\
\hline Posoqueria latifolia (Rudge) Roem. \& Schult. & Av. & & & $\mathbf{x}$ & & V1, V396 \\
\hline Psychotria vellosiana Benth. & Av. & & & $\mathbf{x}$ & & V8 \\
\hline Remijia ferruginea (A. St.-Hil.) DC. & Av. & & & $\mathbf{x}$ & & V606 \\
\hline \multicolumn{7}{|l|}{ SALICACEAE } \\
\hline Xylosma ciliatifolia (Clos) Eichler & Av. & & & $\mathbf{x}$ & & V232 \\
\hline
\end{tabular}


Família/Espécie

SANTALACEAE

Phoradendron crassifolium (Pohl ex DC.) Eichler

SAPINDACEAE

Matayba mollis Radlk.

Serjania gracilis Radlk.

SMILACACEAE

Smilax oblongifolia Pohl ex Griseb.

Smilax sp.

\section{SOLANACEAE}

Athenaea micrantha Sendt.

Aureliana fasciculata (Vell.) Sendtn.

Aureliana velutina Sendt.

Brunfelsia brasiliensis (Spreng.) L.B. Sm. \& Downs

Calibrachoa elegans (Miers) Stehman \& Semir

Schwenkia americana $\mathrm{L}$.

Solanum americanum Mill.

Solanum cladotrichum Vand. ex Dunal

Solanum granuloso leprosum Dunal

Solanum isodynamum Stendt.

Solanum subumbellatum Vell.

Solanum velleum Roem. \& Schult.

STYRACACEAE

Styrax aureus Mart.

TURNERACEAE

Turnera sp.

VELLOZIACEAE

Barbacenia bicolor Mart.

Barbacenia flava Mart. ex Schult. \& Schult. f.

Barbacenia sellovii Goethart \& Henrard

Vellozia albiflora Pohl

Vellozia caruncularis Mart. ex Seub.

Vellozia compacta Mart. ex Schult. \& Schult. f.

Vellozia graminea Pohl

\section{VERBENACEAE}

Lantana fucata Lindl.

Lippia elegans Cham.

Lippia florida Cham.

Lippia gracilis Schauer

Lippia hermannioides Cham.

Lippia lupulina Cham.

Lippia sericea Cham.

Lippia sp.

Stachytarpheta glabra Cham.

VITACEAE

Cissus albida Cambess.

Cissus subrhomboidea (Baker) Planch.

\section{Am. Hábito CC CN CM AA Voucher}

$\begin{array}{lll}\text { P } & \text { x } & \text { L3950 }\end{array}$

$\begin{array}{llll}\text { Av. } & & \mathbf{x} & \text { B147, B151 } \\ \text { Tr. } & \mathbf{x} & \mathbf{x} & \text { V3 }\end{array}$

$\begin{array}{lll}\text { S.Arb. } & \mathbf{x} & \text { B16 }\end{array}$

$\begin{array}{lll}\text { Tr. } & \mathbf{x} & \text { V112 }\end{array}$

$\begin{array}{lll}\text { Arb. } & \mathbf{x} & \text { S2309 }\end{array}$

$\begin{array}{lll}\text { Arb. } & \mathbf{x} & \text { V552 }\end{array}$

$\begin{array}{lll}\text { Arb. } & \mathbf{x} & \text { S2310 }\end{array}$

$\begin{array}{lll}\text { Arb. } & \mathbf{x} & \text { V398 }\end{array}$

$\begin{array}{lllllll}\text { CR } & \text { ET } & \mathbf{x} & \mathbf{x} & & \mathbf{x} & \text { V228 }\end{array}$

$\begin{array}{lllll}\text { S.Arb. } & \mathbf{x} & \mathbf{x} & \mathbf{x} & \text { V602 }\end{array}$

$\begin{array}{lllll}\text { Arb. } & \mathbf{x} & \mathbf{x} & \mathbf{x} & \mathrm{V} 405\end{array}$

Arb. $\quad \mathbf{x} \quad$ V346

$\begin{array}{llllll}\text { Av. } & \mathbf{x} & \mathbf{x} & \mathbf{x} & \mathbf{x} & \text { V234 }\end{array}$

$\begin{array}{lll}\text { Arb. } & \mathbf{x} & \text { V556 }\end{array}$

Arb. $\quad \mathbf{x} \quad$ S2652

$\begin{array}{llll}\text { Arb. } & \mathbf{x} & \mathbf{x} & \text { V120 }\end{array}$

$\begin{array}{lll}\text { Arb. } & \mathbf{x} & \text { V296 }\end{array}$

$\begin{array}{lll}\text { S.Arb. } & \mathbf{x} & \text { BHCB } 80423\end{array}$

ET $\quad \mathbf{x} \quad$ V576

$\begin{array}{lll}\text { S.Arb. } & \mathbf{x} & \text { V385 }\end{array}$

$\begin{array}{lll}\text { S.Arb. } & \mathbf{x} & \text { S2320 }\end{array}$

S.Arb. $\mathbf{x} \quad$ B156

S.Arb. $\mathbf{x} \quad$ S2406

$\begin{array}{llll}\text { Arb. } & \mathbf{x} & \mathbf{x} & \text { V327 }\end{array}$

$\begin{array}{lll}\text { S.Arb. } & \mathbf{x} & \text { S2312 }\end{array}$

$\begin{array}{llll}\text { Arb. } & \mathbf{x} & \mathbf{x} & \text { B5, B140 } \\ \text { Arb. } & \mathbf{x} & & \text { S2410 } \\ \text { S.Arb. } & & \mathbf{x} & \text { L3261 } \\ \text { Arb. } & \mathbf{x} & & \text { V366 } \\ \text { Arb. } & \mathbf{x} & & \text { V99, V395 } \\ \text { Arb. } & & \mathbf{x} & \text { BHCB 80412 } \\ \text { Arb. } & \mathbf{x} & & \text { V419 } \\ \text { Arb. } & & & \text { BHCB 80410 } \\ \text { Arb. } & \mathbf{x} & & \text { V69 }\end{array}$

$\begin{array}{lll}\text { Tr. } & \mathbf{x} & \text { V795 }\end{array}$

$\begin{array}{lll}\text { Tr. } & \mathbf{x} & \mathbf{S} 2317\end{array}$


Tabela 2 - Cinco famílias mais representativas e seus respectivos números de espécies em cada fitofisionomia dos campos rupestres sobre canga, Serra da Calçada, MG.

\begin{tabular}{|c|c|c|c|c|c|c|c|}
\hline \multicolumn{2}{|c|}{ Canga Couraçada } & \multicolumn{2}{|c|}{ Canga Nodular } & \multicolumn{2}{|l|}{ Capões } & \multicolumn{2}{|c|}{ Áreas Antrópicas } \\
\hline Asteraceae & 17 & Asteraceae & 35 & Myrtaceae & 10 & Poaceae & 15 \\
\hline Poaceae & 15 & Poaceae & 33 & Orchidaceae & 10 & Asteraceae & 6 \\
\hline Fabaceae & 10 & Fabaceae & 19 & Melastomataceae & 8 & Solanaceae & 5 \\
\hline Melastomataceae & 9 & Myrtaceae & 12 & Solanaceae & 6 & Bignoniaceae & 3 \\
\hline Apocynaceae & 8 & Malpighiaceae & 10 & Rubiaceae & 5 & Fabaceae & 3 \\
\hline Total & 153 & Total & 210 & Total & 93 & Total & 47 \\
\hline
\end{tabular}

Os campos rupestres sobre conglomerado hematítico, ou canga couraçada, suportam uma vegetação peculiar, com arbustos que aproveitam a escassa matéria orgânica disponível em fendas de rochas, onde também se estabelecem poucas espécies de gramíneas. Espécies rupícolas são marcantes na composição do ambiente. Dentre as espécies arbustivas, que são $34 \%$ das ocorrentes em canga couraçada (Fig. 3), são freqüentes as Asteraceae Lychnophora pinaster, Pseudobrickellia brasiliensis e Symphyopappus brasiliensis, as Euphorbiaceae Croton migrans e Sebastiania glandulosa, as Fabaceae como Bauhinia rufa, Mimosa calodendron e Periandra mediterranea, algumas Verbenaceae como Lippia gracilis, L. hermannioides e Stachytarpheta glabra, além de Diplusodon myrsinitis, Eriope macrostachya, Microlicia pseudoscoparia, Myrsine lancifolia, Ocotea tristis, Peixotoa tomentosa e Vellozia compacta. As ervas terrestres, representadas por poucas espécies de gramíneas, como Andropogon ingratus, Axonopus siccus, Panicum cyanescens, Paspalum scalare e Sporobolus metallicolus, e Cyperaceae, como Bulbostylis fimbriata, Cyperus subcastaneus e Trilepis lhotzkiana, correspondem a $24 \%$ das espécies levantadas em canga couraçada, sendo esta a menor proporção de ervas terrestres dentre os ambientes considerados. Dentre as rupícolas, as diversas espécies de Orchidaceae, como Acianthera teres,
Epidendrum secundum, Oncidium gracile, Oncidium warmingii, Sophronitis caulescens e $S$. crispata se misturam a Bromeliaceae, como Dyckia consimilis e Vriesea minarum, Cactaceae (Arthrocereus glaziovii) e Piperaceae (Peperomia decora e $P$. galliodes). Destacam-se, também, espécies bulbosas como Habranthus irwinianus e Hippeastrum glaucescens, e tuberosas, como Sinningia rupicola. Muitas das espécies encontradas em canga nodular também foram relatadas para outras áreas de campos rupestres da Serra do Espinhaço (Giulietti et al. 1987, Pirani et al. 2003, Stannard 1995).

Pequenos capões de vegetação densa, com predomínio de arvoretas que raramente ultrapassam $5 \mathrm{~m}$ de altura, se difundem ao longo dos trechos de canga couraçada. Notase um sub-bosque bem estabelecido, com elementos herbáceos e arbustivos, e uma freqüência expressiva de poucas espécies epífitas. As espécies arbóreas correspondem à maioria, ou $40 \%$, das espécies nestes capões (Fig. 3). As mais freqüentes são Cabralea canjerana, Clusia arrudae, Copaifera langsdorffii, Guapira obtusata, Guatteria sellowiana, Lafoensia pacari, Matayba mollis, Maytenus gonoclada, Miconia chartacea, Myrcia crassifolia, Posoqueria latifolia, Prunus myrtifolia, Psychotria velloziana, Siphoneugena densiflora e Vitex polygama, todas encontradas também em formações de floresta estacional semidecidual na região do Quadrilátero Ferrífero (Meyer $e t$ 
al. 2004, Sposito \& Stehmann 2006). Notável é o fato que estas espécies, quando vegetam em campos rupestres sobre canga, não alcançam o mesmo porte que se observa em formações de floresta estacional semidecidual na região, onde algumas podem ultrapassar 25 $\mathrm{m}$ de altura (Meyer et al. 2004). Da mesma forma, Porto \& Silva (1989) já relatavam ecótipos com nanismo em vegetação metalófila na Serra de Carajás. Dentre as espécies epífitas encontradas nestes capões, ou $6 \%$ do total neste ambiente, citam-se poucas espécies de bromélias (Aechmea bromeliifolia, Billbergia elegans e Tillandsia recurvata), uma orquídea (Oncidium warmingii) e uma Araceae (Anthurium scandens). No subbosque verificam-se espécies herbáceas, rupícolas e/ou terrestres, como Acianthera limae, Anthurium minarum, Bifrenaria tyrianthina, Coccocypselum lanceolatum, Dichorisandra hexandra, Neomarica rupestris, Oncidium pirarene, Prescottia montana, $P$. oligantha, Zygopetalum mackayi, e algumas arbustivas como Athenaea micrantha, Aureliana velutina, Brunfelsia brasiliensis, Erythroxylum bicolor, Eugenia sonderiana, Hololepis pedunculata, Justicia riparia, Leandra aff. cancellata, Miconia pepericarpa e Ruellia villosa. A maioria das lianas ou trepadeiras levantadas foi verificada nestes capões, onde representam $15 \%$ das espécies. Podem-se citar Banisteriopsis campestris, Bidens brasiliensis, Cissus subrhomboidea, $C$. albida, Coccoloba scandens, Ditassa aequicymosa, Serjania gracilis, Passiflora amethystina e Mandevilla moricandiana, etc.

Nas áreas antropizadas sobre solo ferruginoso, observam-se principalmente espécies ruderais, de ampla distribuição geográfica. Dentre elas, Andropogon bicornis, A. gayanus, Baccharis trimera, Chaptalia integerrima, Chloris pycnothrix, Melinis minutiflora, $M$. repens, Megathyrsus maximus, Hyparrhenia rufa, Pyrostegia venusta, Schwenkia americana,
Setaria parviflora, Sida glaziovii, Solanum americanum e Waltheria indica.

Enfatiza-se que o número de espécies aqui relatado não corresponde à total riqueza de espécies da Serra da Calçada, caracterizada por um mosaico de ambientes distintos. A restrição da área amostral às áreas com solo ferruginoso fornece apenas uma noção parcial da riqueza local, o que limita a realização de comparações florísticas com outras áreas. Nos campos rupestres sobre quartzito, que ocupam grandes extensões da porção oeste da Serra, existe um grande número de espécies aqui não listadas que contribuem de forma importante com a riqueza florística da Serra da Calçada (Viana 2002). Da mesma forma, nos trechos de matas de galeria, bastante freqüentes ao longo dos campos rupestres sobre quartzito, há uma vegetação extremamente diferenciada em relação à encontrada em canga. Nessas matas, se observam diversas espécies arbóreas e epífitas que não foram observadas na vegetação sobre o solo metalífero, como Casearia arborea, Dendropanax cuneatum, Drymis brasiliensis, Geonoma schottiana, Nectandra oppositifolia, Podocarpus sellowi, Oncidium crispum e Sophronitis pumila.

Algumas espécies encontradas são consideradas possivelmente endêmicas dos campos rupestres sobre canga do Quadrilátero Ferrífero. Citam-se Arthrocereus glaziovii (Taylor \& Zappi 2004), Dyckia consimilis, Vriesea minarum (Versieux 2005), Mimosa calodendron (Barneby 1991) e Sinningia rupicola (Chautems, dados não publicados). Esta última, segundo Hoehne (1958), teria ocorrência para o estado de São Paulo, mas de acordo com Chautems (dados não publicados) o exemplar citado como Sinningia rupicola para São Paulo trata-se S. striata (Fritsch) Chautems. A bela solanácea Calibrachoa elegans, restrita, na Serra do Espinhaço, a campos supestres sobre canga, 
apresenta curiosa disjunção com áreas de cerrado no sul de Minas Gerais, contando com um único registro para o município mineiro de Santana do Garambéu (J. R. Stehmann, dados não publicados).

Doze espécies, ou $3,6 \%$ do total, encontram-se na Lista Vermelha das Espécies Ameaçadas de Extinção da Flora de Minas Gerais (Mendonça \& Lins 2000). Dentre elas, sete estão sob o status de vulnerável (Cinnamomum quadrangulum, Ditassa aequicymosa, D. linearis, Guatteria sellowiana, Lychnophora pinaster, Oncidium warmingii e Sinningia rupicola), três em perigo (Camarea hirsuta, Hololepis pedunculata, Ocotea pulchella) e duas criticamente em perigo Arthrocereus glaziovii e Calibrachoa elegans (= Petunia elegans). Estima-se que o número de espécies vegetais realmente ameaçadas de extinção nos campos rupestres sobre canga seja maior que o aqui apresentado, uma vez que a lista publicada pela Fundação Biodiversitas para o Estado (Mendonça \& Lins 2000), apesar de importante, necessita de detalhada revisão. Algumas espécies endêmicas do Quadrilátero Ferrífero e que ocorrem em vegetação de canga não constam na referida lista, como, por exemplo, a orquídea Sophronitis millerii (R. C. Mota, dados não publicados), as bromélias Dyckia consimilis e Vriesea minarum (Versieux 2005), e a pequena erva lactescente Minaria monocoronata (T. U. Konno, dados não publicados).

Os campos rupestres sobre canga de Minas Gerais, que são restritos a pequenas áreas do Quadrilátero Ferrífero, podem ser considerados um ambiente seriamente em perigo. A presença de diversas espécies ameaçadas de extinção e/ou endêmicas associada à sua singularidade abiótica e, principalmente, ao acelerado ritmo de destruição a que estão submetidos, reforça a urgência de preservação de áreas que compreendam esta formação vegetal.

\section{Agradecimentos}

Os autores expressam seus agradecimentos aos pesquisadores J. R. Stehmann e A. Barros pelas importantes coletas e a todos os especialistas que contribuíram na identificação de espécies: M. Sobral (Myrtaceae), J. Nakajima (Asteraceae), A. M. Teles (Asteraceae), J. R. Stehmann (Solanaceae), L. M. Versieux (Bromeliaceae), R. Goldenberg (Miconia e Leandra), R. Mello-Silva (Velloziaceae), F. R. Salimena (Verbenaceae) e R. C. Mota (Orchidaceae). O primeiro autor agradece F. S. F. Leite pela ajuda nas campanhas de campo e aos revisores $a d$ hoc pelas sugestões no texto final.

\section{REFERÊNCIAS BIBLIOGRÁFICAS}

APG. 2003. An update of the Angiosperm Phylogeny Group classification for the orders \& families of flowering plants: APG II. Botanical Journal of the Linnean Society 141: 399-436.

Araújo, A. O.; Souza, V. C. \& Chautems, A. 2005. Gesneriaceae da Cadeia do Espinhaço de Minas Gerais, Brasil. Acta Botanica Brasilica 28(1): 109-135.

Barneby, R. C. 1991. Sensitivae censitae: a description of the genus Mimosa Linneaus (Mimosaceae) in the New World. The New York Bootanical Gardens, New York, 835p.

Brandão, M. 1992. Caracterização geomorfológica, climática, florística e faunística da Serra do Curral em Belo Horizonte, MG. Daphne 2: 13-38.

\& Gavilanes, M. L. 1990. Mais uma contribuição para o conhecimento da Cadeia do Espinhaço em Minas Gerais, Serra da Piedade - II. Daphne 1: 26-43.

; Gavilanes, M. L.; Laca-Buendia, J. P.; Macedo, J. F. \& Cunha, L. H. S. 1991. Contribuição para o conhecimento da Cadeia do Espinhaço em Minas Gerais (Serra de Itabirito) - III. Daphne 1: 39-41. 
Brasil. 1978. Balanço mineral brasileiro: bens minerais selecionados. Ministério das Minas e Energia, 212p.

Drummond, G. M.; Martins, C. S.; Machado, A. B. M; Sebaio, F. A. \& Antonini, Y. 2005. Biodiversidade em Minas Gerais: um atlas para sua conservação. 2a ed. Fundação Biodiversitas, Belo Horizonte, 222p.

Giulietti, A. M.; Harley, R. M.; Queiroz, L. P.; Wanderley, M. G. L. \& Pirani, J. R. 2000. Caracterização e endemismos nos campos rupestres da Cadeia do Espinhaço. In: Cavalcanti, T. B. \& Walter, B. M. T. (org.). Tópicos atuais em botânica. $1^{a}$ ed. SBB/CENARGEN, Brasília. Pp. 311-318.

; Menezes, N. L.; Pirani, J.R.; Meguro, M. \& Wanderley, M. G. L. 1987. Flora da Serra do Cipó, Minas Gerais: Caracterização e Lista das Espécies. Boletim de Botânica da Universidade de São Paulo, 9: 1-151.

; Pirani, J. R. \& Harley, R. M. 1997. Espinhaço Range region. In: Davis, $\mathrm{S}$. D.; Heywood, V. H.; MacBryde, O. H.; Villa-Lobos, J. \& Hamilton, A. C. (eds.). Centers of plant diversity: a guide $\&$ strategy for their conservation. Cambrige. Pp. 397-404.

Grandi, T. S. M.; Siqueira, J. S. \& Paula, J. A. 1988. Levantamento florístico da flora fanerogâmica dos campos rupestres da Serra da Piedade, Caeté, Minas Gerais. Pesquisas - Botânica 39: 89-74.

Harley, R. M. 1995. Flora of the Pico das Almas, Chapada Diamantina-Bahia, Brasil. Stannard, B. L. E. (ed.). The trustees of the Royal Botanic Gardens, Kew, 853p.

\& Mayo, S. J. 1980. Florula of Mucugê, Chapada Diamantina, Brazil. Royal Botanical Gardens, Kew, 227p.

Hoehne, F. C. 1958. Novidades da família das Gesneriaceae do Brasil. Sellowia 9: 37-79.

Larcher, W. 1995. Physiological Plant ecology. Springer Verlag, Berlin, 506p.
Magalhães, G. M. 1966. Sobre os cerrados de Minas Gerais. Anais da Academia Brasileira de Ciências 38(supl.): 59-70.

Meguro, M.; Pirani, J. R.; Giulietti, A. M. \& Mello-Silva, R. 1994. Phytophysiognomy \& composition of the vegetation of Serra do Ambrósio, Minas Gerais, Brazil. Revista Brasileira de Botânica 17: 149-166.

Mendonça, M. P. \& Lins, L. V. 2000. Lista Vermelha das Espécies Ameaçadas de Extinção da Flora de Minas Gerais. Biodiversitas \& Fundação Zoo-Botânica de Belo Horizonte. Belo Horizonte, 157p.

Mendonça, R. C.; Felfili, J. M.; Walter, B. M. T.; Silva Júnior, M. C.; Rezende, A. V.; Filgueiras, T. S. \& Nogueira, P.E. 1998. Flora Vascular do Cerrado. In: Sano, S. M. \& Almeida, S. P. Cerrado ambiente e flora., EMBRAPA-CPAC, Planaltina. Pp. 289-556.

Meyer, S. T.; Silva, A. F.; Júnior, P. M. \& MeiraNeto, J. A. A. 2004. Composição florística da vegetação arbórea de um trecho de floresta de galeria do Parque Estadual do Rola-Moça na Região Metropolitana de Belo Horizonte, MG, Brasil. Acta Botânica Brasílica 18(4): 701-709.

Morelato, P. C. \& Rosa, N. A. 1991. Caracterização de alguns tipos de vegetação na região amazônica, Serra dos Carajás, Pará, Brasil. Revista Brasileira de Botânica 14: 1-14.

Nakajima, J. N. \& Semir, J. 2001. Asteraceae do Parque Nacional da Serra da Canastra, Minas Gerais, Brasil. Revista Brasileira de Botânica 24(4): 471-478.

Pirani, J. R.; Giulietti, A, M.; Mello-Silva, R. \& Meguro, M. 1994. Checklist \& Patterns of Geographic Distribution of the Vegetation of Serra do Ambrósio, Minas Gerais, Brazil. Revista Brasileira de Botânica 17: 133-147.

; Mello-Silva, R \& Giulietti, A. M. 2003. Flora de Grão-Mogol, Minas Gerais. Boletim de Botânica da Universidade de São Paulo 21(1): 1-27. 
Porto, M. L. \& Silva, M. F. F. 1989. Tipos de vegetação metalófila em áreas da Serra de Carajás e de Minas Gerais. Acta Botanica Brasilica 3: 13-21.

Rizzini, C. T. 1979. Tratado de fitogeografia do Brasil. Aspectos sociológicos e florísticos. HUCITEC/EDUSP, São Paulo, 374p.

Rodela, L. G. 1998. Cerrados de altitude e campos rupestres do Parque Estadual do Ibitipoca, sudeste de Minas Gerais: distribuição e florística por subfisionomia da vegetação. Revista do Departamento de Geografia 12: 163-189.

Romero, R \& Martins, A. B. 2001. Melastomataceae do Parque Nacional da Serra da Canastra, Minas Gerais, Brasil. Revista Brasileira de Botânica 25(1): 19-24.

Secco, R. S \& Mesquita, A. I. 1983. Notas sobre a vegetação de canga na Serra Norte - I. Boletim do Museu Paraense Emílio Goeldi, série Botânica 59: 1-13.

Silva, M. F. F. 1991. Análise florística da vegetação que cresce sobre canga hematítica em Carajás - Pará (Brasil). Boletim do Museu paraense Emílio Goeldi, série Botânica 7: 79-105.

1992. Distribuição de matais pesados na vegetação metalófila de Carajás. Acta Botanica Brasilica 6: 107-122.

Spósito, T. C. \& Stehmann, J. R. 2006. Heterogeneidade florística e estrutural deremanescentes florestais da Área de Proteção Ambiental ao Sul da Região Metropolitana de Belo Horizonte (APA Sul-RMBH), Minas Gerais, Brasil. Acta Botanica Brasilica (20)2: 347-362.

Stannard, B. L. 1995. Flora of the Pico das Almas: Chapada Diamantina - Bahia, Brazil. Royal Botanical Gardens, Kew, 853p.
Taylor, N. P \& Zappi, D. C. 2004. Cacti of Eastern Brazil. Royal Botanical Gardens, Kew, 499p.

Teixeira, W. A \& Lemos-Filho, J. P. 1998. Metais pesados em folhas de espécies lenhosas colonizadoras de uma área de mineração de ferro em Itabirito, Minas Gerais. Árvore 22: 381-388.

Teixeira W. A \& Lemos-Filho, J. P. 2002. Fatores edáficos e a colonização de espécies lenhosas em uma cava de mineração de ferro em Itabirito, Minas Gerais. Árvore 26: 25-33.

Versieux, L. M. 2005. Bromeliáceas de Minas Gerais: catálogo, distribuição geográfica e conservação. Dissertação de Mestrado. Universidade Federal do Rio de Janeiro, Rio de Janeiro, 234p.

Viana, P. L. 2002. Levantamento das plantas vasculares em uma área de formações campestre na Serra da Moeda, MG. Monografia de Bacharelado. Universidade Federal de Minas Gerais. Belo Horizonte, 23p.

Vincent, R. C. 2004. Florística, fitossociologia e relações entre a vegetação e o solo em área de campos ferruginosos no Quadrilátero Ferrífero, Minas Gerias. Tese de doutorado. Universidade de São Paulo, São Paulo, 145p.

; Jacobi, C. M. \& Antonini, Y. 2002. Diversidade na adversidade. Ciência Hoje 31(185): 64-67.

Zappi, D. C.; Lucas, E.; Stannard, B. L.; Lughadha, E. N.; Pirani, J. R.; Queiroz, L. P.; Atkins, S.; Hind, D. J. N.; Giulietti, A. M.; Harley; R. M.; Carvalho, A. M. 2003. Lista das plantas vasculares de Catolés, Chapada Diamantina, Bahia. Boletim de Botânica da Universidade de São Paulo 21(2): 345-398. 